\title{
Transition metal-promoted synthesis of 2-aryl/heteroaryl-thioquinazoline: C-S Bond formation by "Chan-Lam Cross-Coupling" Reaction
}

\author{
SATYA KARUNA PULAKHANDAM ${ }^{\mathrm{a}}$, NARESH KUMAR KATARI ${ }^{\mathrm{b}, *}$ (D) \\ RAVI PRAKASH REDDY MANDA ${ }^{\mathrm{c}}$ \\ a Jawaharlal Nehru Technological University Kakinada, Kakinada 533 003, Andhra Pradesh, India \\ ${ }^{\mathrm{b}}$ Department of Chemistry, GITAM School of Technology, GITAM University, HTP campus, Rudraram, \\ Medak 502 329, Telangana, India \\ ${ }^{\mathrm{c}} \mathrm{C}-\mathrm{MET}$, IDA Phase II, Cherlapally, HCL (PO), Hyderabad 500 051, Telangana, India \\ Email: karunasathya@gmail.com; dr.n.k.katari@gmail.com; mrpreddy@cmet.gov.in
}

MS received 16 September 2016; revised 30 November 2016; accepted 30 November 2016

\begin{abstract}
An efficient method for the synthesis of $S$-aryl/heteroaryl-quinazoline has been developed through the cross-coupling of 1,4-dihydroquinazoline with a variety of aryl and heteroaryl boronic acids assisted by $\left[\mathrm{Cu}(\mathrm{OAc})_{2}\right]$ as the catalyst for the formation of carbon-sulfur bonds. This newly developed method demonstrates that the conditions of the traditional copper-catalyzed Chan-Lam reaction can be improved. Optimized reaction involves base, solvent and catalyst.
\end{abstract}

Keywords. Chan-Lam coupling; quinazoline; boronic acids; copper acetate.

\section{Introduction}

The quinazoline skeleton, when selectively functionalized, is a building block for the preparation of numerous alkaloids and substances with pronounced biological activities. ${ }^{1}$ Quinazoline is the core ring of numerous drug compounds, the scaffold of which always includes a thiol substituent at position-2 of the quinazoline ring.

Transition-metal-catalyzed carbon-heteroatom crosscoupling reactions represent great contribution to the recent growth of organic synthesis. ${ }^{2}$ Although aryl sulfides have broad application in the pharmaceutical industry and material science ${ }^{3}$ and as intermediates in organic synthesis, ${ }^{4}$ the formation of carbon-sulfur bonds has received less attention. Difficulties in C-S bond formation can be attributed to the sulfur species rapidly and irreversibly deactivating the catalyst. ${ }^{5}$ So the efficient formation of the C-S bond is one of the most important aspect of organic chemistry.

\section{Experimental}

\subsection{Materials and Methods}

All the reagents and chemicals were procured from commercial sources (SD Fine Chemicals, India; Aldrich) and used without any further purification. Melting points are

*For correspondence uncorrected and were determined in open capillary tubes in melting point apparatus. TLC was performed on silica gel$\mathrm{G}$ and spotting was done using iodine/ $\mathrm{KMnO}_{4}$ staining or UV light. IR spectra were recorded with Perkin-Elmer 1000 instrument in $\mathrm{KBr}$ phase. ${ }^{1} \mathrm{H}$ NMR was recorded on VARIAN $300 \mathrm{MHz}$ instrument and Mass spectra were recorded on Agilent-LC-MS instrument. The following experimental procedures are representative of the general procedures used to synthesize all compounds.

\subsection{Synthesis of the compounds}

Compounds a to $\mathbf{r}$ were prepared by following a general synthetic procedure in which the solution of 1,4dihydroquinazoline-2-thiol $(100 \mathrm{mg}, 0.609 \mathrm{mmol})$ in dichloromethane $(4 \mathrm{~mL})$ was added the $\mathrm{Cu}(\mathrm{OAc})_{2}(166 \mathrm{mg}$, $0.913 \mathrm{mmol}), \mathrm{Et}_{3} \mathrm{~N}(0.43 \mathrm{~mL}, 3.045 \mathrm{mmol})$ and stirred for 10-15 min at RT, followed by addition of substituted boronic acid $(1.219 \mathrm{mmol})$. The reaction mixture was stirred at RT for $12 \mathrm{~h}$. After completion of the reaction, as indicated by TLC, the reaction mixture was quenched with water $(10 \mathrm{~mL})$ and extracted with dichloromethane $(2 \times 15 \mathrm{~mL})$. The organic layer was washed with water, brine solution and dried over anhydrous $\mathrm{Na}_{2} \mathrm{SO}_{4}$, filtered the solvent, and concentrated under reduced pressure to give the crude compound. The crude compound was purified by flash column chromatography (100-200 mesh silica gel), eluted at 10-20\% ethyl acetate/pet ether to afford the $S$-substituted quinazoline derivative. The characterization data for the compounds are given below. 
2.2.1 2-(p-tolylthio) quinazoline (Table 4, Entry a): Pale yellow solid. [Yield: $130 \mathrm{mg}, 85 \%$ ] $\mathrm{R}_{f}: 0.7,8 \% \mathrm{EtOAc} / \mathrm{Pet}$ ether; Analysis: LC-MS: $m / z \mathrm{C}_{15} \mathrm{H}_{12} \mathrm{~N}_{2} \mathrm{~S}$ for $(\mathrm{M}+\mathrm{H})$ : Calculated: 252.3; Found, 253.3 (82.3\%). IR/ $\mathrm{cm}^{-1}$ : 1796.91, $1615.00,1548.45,1483.60,1439.43,1369.55,1216.85$, 1159.01, 1094.48, 968.86, 863.36, 777.29, 757.23, 688.92 and 660.43. ${ }^{1} \mathrm{H} \mathrm{NMR}\left(\mathrm{CDCl}_{3}, 400 \mathrm{MHz}\right): 9.15(1 \mathrm{H}, \mathrm{s})$, 7.83-7.77 (3H, m), 7.53-7.50 (3H, m), $7.35(1 \mathrm{H}, \mathrm{t}, J=7.6 \mathrm{~Hz})$, $7.25(1 \mathrm{H}, \mathrm{d}, J=2.0 \mathrm{~Hz}), 2.40(3 \mathrm{H}, \mathrm{s}) .{ }^{13} \mathrm{C} \mathrm{NMR}\left(\mathrm{CDCl}_{3}\right.$, $300 \mathrm{MHz}$ ): 160.63, 150.96, 138.90, 135.45, 134.47, 132.07, $129.92,129.63,128.93,127.31,126.53,122.61,105.0,22.07$ ppm. MR: $50-53^{\circ} \mathrm{C}$.

2.2.2 2-Allylsulfanyl-quinazoline (Table 4, Entry b): Yellow thick mass. [Yield: $95 \mathrm{mg}, 77 \%$ ] $\mathrm{R}_{f}$ : 0.6, 8\% EtOAc/Pet ether; Analysis: LC-MS: $m / z \mathrm{C}_{11} \mathrm{H}_{10} \mathrm{~N}_{2} \mathrm{~S}$ for $(\mathrm{M}+\mathrm{H})$ : Calculated: 202.06; Found, 202.99 (99.64\%). IR/ $\mathrm{cm}^{-1}$ : 1709.17, $1615.84,1559.81,1549.91,1485.23,1370.78,1219.10$, 1164.34, 1134.86, 1100.05, 918.46, 755.83 and 691.90. ${ }^{1} \mathrm{H}$ NMR $\left(\mathrm{CDCl}_{3}, 400 \mathrm{MHz}\right): 9.14(1 \mathrm{H}, \mathrm{s}), 7.84-7.80(3 \mathrm{H}, \mathrm{m})$, 7.53-7.49 (1H, m), 6.10-6.03 (1H, m), $5.38(1 \mathrm{H}, \mathrm{dd}, J=$ $17.2,1.0 \mathrm{~Hz}), 5.15(1 \mathrm{H}, \mathrm{d}, J=9.6 \mathrm{~Hz}), 3.96(2 \mathrm{H}, \mathrm{d}, J=7.2$ $\mathrm{Hz}) .{ }^{13} \mathrm{C} \mathrm{NMR}\left(\mathrm{CDCl}_{3}, 400 \mathrm{MHz}\right): 167.72,160.16,150.82$, $134.41,133.57,127.37,126.93,126.25,122.38,117.77$, 67.04 ppm.

2.2.3 2-(4-Isopropyl-phenylsulfanyl)-quinazoline (Table 4, Entry c): Pale Yellow thick mass [Yield: $147 \mathrm{mg}, 87 \%$ ] $\mathrm{R}_{f}$ : $0.7, \quad 10 \%$ EtOAc/Pet ether; Analysis: LC-MS: $m / z \quad \mathrm{C}_{17} \mathrm{H}_{16} \mathrm{~N}_{2} \mathrm{~S}$ for $(\mathrm{M}+\mathrm{H})$ : Calculated: 280.10; Found, 281.31 (83\%). IR/cm ${ }^{-1}: 1733.29,1616.78,1579.22,1547.95$, $1370.14,1218.33,1160.81,1095.79,866.12,754.49,695.67$ and 466.67. ${ }^{1} \mathrm{H} \mathrm{NMR}\left(\mathrm{CDCl}_{3}, 400 \mathrm{MHz}\right): 9.16(1 \mathrm{H}, \mathrm{s}), 7.83-$ $7.77(3 \mathrm{H}, \mathrm{m}), 7.51(2 \mathrm{H}, \mathrm{t}, J=7.4 \mathrm{~Hz}), 7.39-7.36(2 \mathrm{H}, \mathrm{m})$, $7.30(1 \mathrm{H}, \mathrm{d}, J=8.0 \mathrm{~Hz}), 2.98-2.94(1 \mathrm{H}, \mathrm{m}), 1.56(6 \mathrm{H}, \mathrm{d}$, $J=8.0 \mathrm{~Hz}) .{ }^{13} \mathrm{C} \mathrm{NMR}\left(\mathrm{CDCl}_{3}, 300 \mathrm{MHz}\right): 160.82,151.17$, $145.35,134.67,134.51,132.34,129.91,129.17,128.91$, $127.50,126.73,122.83,34.67,29.88,28.91 \mathrm{ppm}$.

2.2.4 2-(pyridin-4-ylsulfanyl)-quinazoline (Table 4, Entry d): Off white solid [Yield: $125 \mathrm{mg}, 86 \%$ ] $\mathrm{R}_{f}$ : $0.2,20 \%$ EtOAc/Pet ether; Analysis: LC-MS: $m / z \quad \mathrm{C}_{13} \mathrm{H}_{9} \mathrm{~N}_{3} \mathrm{~S}$ for $(\mathrm{M}+\mathrm{H})$ : Calculated: 239.05; Found, 239.89 (94.51\%); IR/cm ${ }^{-1}$ : 1734.59, 1610.63, 1555.78, 1465.06, 1367.47, 1215.38, 1154.65, 1123.18, 1091.58, 873.12, 789.83, 765.85, 705.15 and $689.89 .{ }^{1} \mathrm{H} \mathrm{NMR}\left(\mathrm{CDCl}_{3}, 400 \mathrm{MHz}\right): 9.16$ $(1 \mathrm{H}, \mathrm{s}), 8.8(2 \mathrm{H}, \mathrm{s}), 8.04(1 \mathrm{H}, \mathrm{d}, J=8.0 \mathrm{~Hz}), 7.83(2 \mathrm{H}, \mathrm{t}$, $J=7.6 \mathrm{~Hz}), 7.75(1 \mathrm{H}, \mathrm{d}, J=8.4 \mathrm{~Hz}), 7.54(1 \mathrm{H}, \mathrm{t}, J=$ $7.80 \mathrm{~Hz}), 7.45(1 \mathrm{H}, \mathrm{s}) .{ }^{13} \mathrm{C} \mathrm{NMR}\left(\mathrm{CDCl}_{3}, 400 \mathrm{MHz}\right): 167.03$, $160.71,150.87,134.69,127.34,127.28,126.88,122.82 \mathrm{ppm}$. MR: $128-131^{\circ} \mathrm{C}$.

2.2.5 2-(3-Methoxy-phenylsulfanyl)-quinazoline (Table 4, Entry e): Pale yellow solid [Yield: $146 \mathrm{mg}, 90 \%$ ] $\mathrm{R}_{f}: 0.7$, $10 \%$ EtOAc/Pet ether; Analysis: LC-MS: $m / z \mathrm{C}_{15} \mathrm{H}_{12} \mathrm{~N}_{2} \mathrm{OS}$ for $(\mathrm{M}+\mathrm{H})$ : Calculated: 268.07; Found, 269.10 (98.49\%); $\mathrm{IR} / \mathrm{cm}^{-1}: 1612.24,1577.80,1556.60,1477.28,1374.57$, $1271.7,1246.72,1165.47,1097.34,1070.97,1022.92$, 790.26, 756.38 and 690.53. ${ }^{1} \mathrm{H}$ NMR (DMSO-d 6 , $\left.300 \mathrm{MHz}\right)$ : $9.36(1 \mathrm{H}, \mathrm{s}), 8.04(1 \mathrm{H}, \mathrm{d}, J=8.10 \mathrm{~Hz}), 7.92(1 \mathrm{H}, \mathrm{t}, J=$ $7.05 \mathrm{~Hz}), 7.69-7.60(3 \mathrm{H}, \mathrm{m}), 7.54-7.48(1 \mathrm{H}, \mathrm{m}), 7.17(1 \mathrm{H}, \mathrm{d}$, $J=8.40 \mathrm{~Hz}), 7.05(1 \mathrm{H}, \mathrm{t}, J=7.50 \mathrm{~Hz}), 3.7(3 \mathrm{H}, \mathrm{s}) .{ }^{13} \mathrm{C}$ NMR $\left(\mathrm{CDCl}_{3}, 400 \mathrm{MHz}\right): 168.09,160.35,159.98,150.94$, $136.78,134.25,131.22,127.23,127.20,126.28,122.47$, $120.99,118.23,111.57,55.92$ ppm. MR: $115-118^{\circ} \mathrm{C}$.

2.2.6 2-(3-Nitro-phenylsulfanyl)-quinazoline (Table 4, Entry f): Yellow solid [Yield: $152 \mathrm{mg}, 88 \%$ ] $\mathrm{R}_{f}$ : 0.6, 12\% EtOAc/Pet ether; Analysis: LC-MS: $m / z \mathrm{C}_{14} \mathrm{H}_{9} \mathrm{~N}_{3} \mathrm{O}_{2} \mathrm{~S}$ for $(\mathrm{M}+\mathrm{H})$ : Calculated: 283.04; Found, 284.0 (96.58\%); IR/ $\mathrm{cm}^{-1}: 1615.72$, $1595.94,1551.74,1506.14,1339.60,1219.04,1160.58$, $1094.35,1084.34,845.68,753.10,741.87,730.40$ and 685.89. ${ }^{1} \mathrm{H} \mathrm{NMR}\left(\mathrm{CDCl}_{3}, 400 \mathrm{MHz}\right): 9.20(1 \mathrm{H}, \mathrm{s}), 8.28$ $(2 \mathrm{H}, \mathrm{d}, J=3.60 \mathrm{~Hz}), 7.92-7.86(4 \mathrm{H}, \mathrm{m}), 7.80(1 \mathrm{H}, \mathrm{d}$, $J=8.80 \mathrm{~Hz}), 7.61-7.57(1 \mathrm{H}, \mathrm{m}) .{ }^{13} \mathrm{C} \mathrm{NMR}\left(\mathrm{CDCl}_{3}\right.$, $300 \mathrm{MHz})$ : 160.8, 151.17, 145.35, 134.67, 134.51, 132.34, 129.91, 129.18, 128.91, 127.5, 126.73, 122.83, 110.2. MR: $170-173^{\circ} \mathrm{C}$.

2.2.7 5-(quinazolin-2-ylthio)thiophen-2-carbaldehyde (Table 4, Entry g): Pale yellow solid [Yield: $152 \mathrm{mg}$, 92\%] $\mathrm{R}_{f}$ : $0.5,15 \%$ EtOAc/Pet ether; Analysis: LC-MS: $m / z \mathrm{C}_{13} \mathrm{H}_{8} \mathrm{~N}_{2} \mathrm{OS}_{2}$ for $(\mathrm{M}+\mathrm{H})$ : Calculated: 272.01; Found, 272.75 (92.29\%); IR/ $\mathrm{cm}^{-1}$ : 2924.51, 2853.80, 2676.12, $1742.58,1655.34,1615.65,1464.92,1413.14,1369.13$, $1221.49,1163.32,1096.19,1017.36,799.83,753.55$ and 660.41. ${ }^{1} \mathrm{H} \mathrm{NMR}\left(\mathrm{CDCl}_{3}, 400 \mathrm{MHz}\right): 9.92(1 \mathrm{H}, \mathrm{s}), 9.22$ $(1 \mathrm{H}, \mathrm{s}), 7.91-7.87(3 \mathrm{H}, \mathrm{m}), 7.76(1 \mathrm{H}, \mathrm{d}, J=3.0 \mathrm{~Hz})$, $7.62-7.58(1 \mathrm{H}, \mathrm{m}), 7.43(1 \mathrm{H}, \mathrm{t}, J=2.85 \mathrm{~Hz})$. MR: $92-96^{\circ} \mathrm{C}$.

2.2.8 2-(benzo[b]thiophen-2-ylthio)quinazoline (Table 4, Entry $h$ ): Yellow solid [Yield: $147 \mathrm{mg}, 82 \%$ ] $\mathrm{R}_{f}$ : 0.4, $17 \%$ EtOAc/Pet ether; Analysis: LC-MS: $m / z \mathrm{C}_{16} \mathrm{H}_{10} \mathrm{~N}_{2} \mathrm{~S}_{2}$ for $(\mathrm{M}+\mathrm{H})$ : Calculated: 294.03; Found, 294.81 (85.65\%); $\mathrm{IR} / \mathrm{cm}^{-1}$ : 1738.12, 1615.94, 1572.95, 1555.68, 1456.52, 1367.47, 1168.10, 1109.49, 964.14, 838.87, 746.13, 723.83 and 556.93. ${ }^{1} \mathrm{H} \mathrm{NMR}\left(\mathrm{CDCl}_{3}, 400 \mathrm{MHz}\right): 9.19(1 \mathrm{H}, \mathrm{s})$, 7.85-7.81 (6H, m), 7.66 (1H, s), 7.57-7.53 (1H, m), 7.40$7.37(1 \mathrm{H}, \mathrm{m}) .{ }^{13} \mathrm{C} \mathrm{NMR}\left(\mathrm{CDCl}_{3}, 400 \mathrm{MHz}\right): 167.36,160.84$, $150.96,143.61,139.31,134.74,132.46,129.91,127.36$, $125.14,124.34,124.01,122.78,122.11 \mathrm{ppm}$. MR: $139-143^{\circ} \mathrm{C}$.

2.2.9 2-((2,3-dichlorophenyl)thio)quinazoline (Table 4, Entry i): Off white solid [Yield: $162 \mathrm{mg}, 87 \%$ ] $\mathrm{R}_{f}: 0.7,10 \%$ EtOAc/Pet ether; Analysis: LC-MS: $m / z \mathrm{C}_{14} \mathrm{H}_{8} \mathrm{Cl}_{2} \mathrm{~N}_{2} \mathrm{~S}$ for $(\mathrm{M}+\mathrm{H}, \mathrm{M}+3 \mathrm{H})$ : Calculated: 305.98; Found, 307.0, 308.9 (92.36\%); IR/cm ${ }^{-1}: 1733.89,1623.19,1456.97,1384.47$, 1370.21, 1263.66, 1093.34, 752.77, 611.82 and 497.49. ${ }^{1} \mathrm{H}$ $\mathrm{NMR}\left(\mathrm{CDCl}_{3}, 400 \mathrm{MHz}\right): 9.17$ (1H, s), 7.87-7.78 (4H, m), 7.57-7.53 (3H, m). ${ }^{13} \mathrm{C} \mathrm{NMR}\left(\mathrm{CDCl}_{3}, 400 \mathrm{MHz}\right): 162.24$, 
$160.98,151.07,136.55,134.97,134.25,133.69,133.06$, $130.95,130.23,127.57,127.47,127.14,122.97$ ppm. MR: $119-122^{\circ} \mathrm{C}$.

2.2.10 2-(Quinolin-3-ylsulfanyl)-quinazoline (Table 4, Entry j): Off white solid [Yield: $146 \mathrm{mg}, 83 \%$ ] $\mathrm{R}_{f}: 0.2,10 \%$ EtOAc/Pet ether; Analysis: LC-MS: $m / z \quad \mathrm{C}_{17} \mathrm{H}_{11} \mathrm{~N}_{3} \mathrm{~S}$ for $(\mathrm{M}+\mathrm{H})$ : Calculated: 289.07; Found, 290.15 (98.58\%); $\mathrm{IR} / \mathrm{cm}^{-1}$ : $1616.17,1579.18,1548.27,1486.28,1440.35$, 1370.07, 1218.78, 1162.53, 1097.67, 879.43, 865.32, 830.59, 790.89, 754.48, and 689.64. ${ }^{1} \mathrm{H} \mathrm{NMR}\left(\mathrm{CDCl}_{3}, 400 \mathrm{MHz}\right)$ : $9.11(1 \mathrm{H}, \mathrm{s}), 8.98(1 \mathrm{H}, \mathrm{dd}, J=4.4,1.6 \mathrm{~Hz}), 8.21-8.00$ $(3 \mathrm{H}, \mathrm{m}), 7.98(1 \mathrm{H}, \mathrm{dd}, J=8.8,6.4 \mathrm{~Hz}), 7.85-7.74(3 \mathrm{H}, \mathrm{m})$, 7.56-7.52 (1H, m), 7.48-7.44 (1H, m). ${ }^{13} \mathrm{C} \mathrm{NMR}\left(\mathrm{CDCl}_{3}\right.$, $300 \mathrm{MHz})$ : 160.9, 151.39, 151.12, 148.34, 136.26, 135.89, $134.86,133.93,130.19,129.0,128.76,127.55,127.47$, 127.0, 122.94, 121.76 ppm. MR: $130-133^{\circ} \mathrm{C}$.

2.2.11 2-Phenethylsulfanyl-quinazoline (Table 4, Entry k): Yellow thickmass [Yield: $146 \mathrm{mg}, 90 \%$ ] $\mathrm{R}_{f}: 0.7,8 \%$ EtOAc/Pet ether; Analysis: LC-MS: $m / z \quad \mathrm{C}_{16} \mathrm{H}_{14} \mathrm{~N}_{2} \mathrm{~S}$ for $(\mathrm{M}+\mathrm{H})$ : Calculated: 266.09; Found, 267.08 (98.96\%). $\mathrm{IR} / \mathrm{cm}^{-1}: 1734.64,1616.51,1550.10,1484.23,1370.08$, $1275.41,1261.54,1164.36,1100.69,1035.59,791.14,750.90$ and 698.46. ${ }^{1} \mathrm{H} \mathrm{NMR}\left(\mathrm{CDCl}_{3}, 400 \mathrm{MHz}\right): 9.16(1 \mathrm{H}, \mathrm{s}), 7.89-$ $7.82(3 \mathrm{H}, \mathrm{m}), 7.54-7.49$ (1H, m), 7.35-7.30 (4H, m), 7.25-7.21 $(1 \mathrm{H}, \mathrm{m}), 3.51(2 \mathrm{H}, \mathrm{t}, J=9.6 \mathrm{~Hz}), 3.13(2 \mathrm{H}, \mathrm{t}, J=7.6 \mathrm{~Hz})$. ${ }^{1} \mathrm{H}$ NMR (DMSO-d $6,400 \mathrm{MHz}$ ): 167.12, 161.04, 150.01, 140.32 , 135.07, 128.59, 128.36, 128.12, 126.59, 126.27, $126.22,122.10,48.57,34.85 \mathrm{ppm}$.

2.2.12 2-((3-bromophenyl)thio) quinazoline (Table 4, Entry l): Yellow thick mass [Yield: $158 \mathrm{mg}, 82 \%$ ] $\mathrm{R}_{f}$ : $0.8,5 \%$ EtOAc/Pet ether; Analysis: LC-MS: $m / z \quad \mathrm{C}_{14} \mathrm{H}_{9} \mathrm{BrN}_{2} \mathrm{~S}$ for $(\mathrm{M}+\mathrm{H}, \mathrm{M}+3 \mathrm{H})$ : Calculated: 315.97; Found, 316.75, 318.76 (97.34\%). IR/ $\mathrm{cm}^{-1}: 1741.93,1614.70,1557.78,1446.96$, $1370.79,1219.32,1161.27,1095.68,1018.49$, 969.06, 865.47 and $750.85 .{ }^{1} \mathrm{H} \mathrm{NMR}\left(\mathrm{CDCl}_{3}, 400 \mathrm{MHz}\right): 9.15(1 \mathrm{H}$, s), 7.85-7.74 (4H, m), 7.55-7.51 (2H, m), 7.42-7.38 (1H, m), 7.34-7.29 (1H, m). ${ }^{13} \mathrm{C} \mathrm{NMR}\left(\mathrm{CDCl}_{3}, 300 \mathrm{MHz}\right)$ : 160.72, $151.0,137.42,134.55,133.58,131.53,130.78,130.59$, $127.95,127.33,126.70,122.63,109.99 \mathrm{ppm}$.

2.2.13 2-(4-Trifluoromethoxy-phenylsulfanyl)-quinazoline (Table 4, Entry $\mathrm{m}$ ): Yellow oil [Yield: $149 \mathrm{mg}, 76 \%$ ] $\mathrm{R}_{f}$ : $0.5,15 \%$ EtOAc/Pet ether; Analysis: LC-MS: $m / z \mathrm{C}_{15} \mathrm{H}_{9} \mathrm{~F}_{3} \mathrm{~N}_{2} \mathrm{OS}$ for $(\mathrm{M}+\mathrm{H})$ : Calculated: 322.04; Found, 323.0 (90.42\%). IR/ $\mathrm{cm}^{-1}: 1621.7,1579.88,1559.74$, $1371.17,1258.38,1222.55,1162.16,1096.13,789.31$ and 756.04. ${ }^{1} \mathrm{H}$ NMR $\left(\mathrm{CDCl}_{3}, 300 \mathrm{MHz}\right): 9.18(1 \mathrm{H}, \mathrm{s}), 7.86-$ $7.82(2 \mathrm{H}, \mathrm{m}), 7.76(1 \mathrm{H}, \mathrm{d}, J=8.4 \mathrm{~Hz}), 7.64(2 \mathrm{H}, \mathrm{t}, J=$ $12.4 \mathrm{~Hz}), 7.57-7.52(1 \mathrm{H}, \mathrm{m}), 7.47(1 \mathrm{H}, \mathrm{t}, J=8.1 \mathrm{~Hz})$, $7.30(1 \mathrm{H}, \mathrm{s}) .{ }^{13} \mathrm{C} \mathrm{NMR}\left(\mathrm{CDCl}_{3}, 300 \mathrm{MHz}\right): 167.11,160.74$, $158.4,150.87,139.26,134.72,132.64,130.0,129.1,127.34$, $125.6,122.2,121.7,115.0,111.2 \mathrm{ppm}$.
2.2.14 2-(Furan-2-ylsulfanyl)-quinazoline (Table 4, Entry n): Yellow thick mass [Yield: $100 \mathrm{mg}, 72 \%$ ] $\mathrm{R}_{f}$ : 0.5, $15 \%$ EtOAc/Pet ether; Analysis: LC-MS: $m / z \mathrm{C}_{12} \mathrm{H}_{8} \mathrm{~N}_{2} \mathrm{OS}$ for $(\mathrm{M}+\mathrm{H})$ : Calculated: 228.04; Found, 229.0 (90.13\%). $\mathrm{IR} / \mathrm{cm}^{-1}: 1618.12,1579.39,1549.90,1372.06,1275.19$, $1260.71,1162.74,1098.89,1010.64,868.12,750.70$ and 486.79. ${ }^{1} \mathrm{H} \mathrm{NMR}\left(\mathrm{CDCl}_{3}, 400 \mathrm{MHz}\right): 9.16(1 \mathrm{H}, \mathrm{s}), 8.22(1 \mathrm{H}, \mathrm{s})$, 7.92-7.83 (2H, m), 7.81-7.73 (2H, m), 7.55-7.50 (2H, m). ${ }^{13} \mathrm{C} \mathrm{NMR}\left(\mathrm{CDCl}_{3}, 300 \mathrm{MHz}\right): 167.39,159.94,150.29$, $133.89,131.72,129.52,127.74,126.66,125.95,125.25$, $124.72,121.94 \mathrm{ppm}$.

2.2.15 2-((3-(trifluoromethyl)phenyl)thio)quinazoline (Table 4, Entry o): Yellow thick mass [Yield: $149 \mathrm{mg}, 80 \%$ ] $\mathrm{R}_{f}: 0.6$, 12\% EtOAc/Pet ether; Analysis: LC-MS: $m / z \mathrm{C}_{15} \mathrm{H}_{9} \mathrm{~F}_{3} \mathrm{~N}_{2} \mathrm{~S}$ for $(\mathrm{M}+\mathrm{H})$ : Calculated: 306.04; Found, 307.2 (92.60\%). $\mathrm{IR} /\left(\mathrm{cm}^{-1}\right): 1579.88,1559.74,1371.17,1258.38,1222.55$, 1162.16, 1096.13, 789.31, 756.04 and 702.8. ${ }^{1} \mathrm{H}$ NMR $\left(\mathrm{CDCl}_{3}, 400 \mathrm{MHz}\right): 9.17(1 \mathrm{H}, \mathrm{s}), 8.01(1 \mathrm{H}, \mathrm{s}), 7.76(1 \mathrm{H}, \mathrm{d}$, $J=8.8 \mathrm{~Hz}), 7.69(1 \mathrm{H}, \mathrm{d}, J=8.0 \mathrm{~Hz}), 7.59-7.53(2 \mathrm{H}, \mathrm{m})$, $7.35(1 \mathrm{H}, \mathrm{t}, J=8.0 \mathrm{~Hz}), 7.76(1 \mathrm{H}, \mathrm{d}, J=7.6 \mathrm{~Hz}), 7.00(1 \mathrm{H}$, $\mathrm{d}, J=8.4 \mathrm{~Hz}) .{ }^{13} \mathrm{C} \mathrm{NMR}\left(\mathrm{CDCl}_{3}, 300 \mathrm{MHz}\right): 160.7,155.2$, 150.6, 134.7, 132.6, 132.7, 131.8, 129.4, 129.3, 128.4, 127.6, $126.5,125.7,122.7,119.2 \mathrm{ppm}$.

2.2.16 2-(naphthalen-2-ylthio) quinazoline (Table 4, Entry p): Colorless oil [Yield: $158 \mathrm{mg}, 90 \%$ ] $\mathrm{R}_{f}: 0.6,10 \%$ EtOAc/Pet ether; Analysis: LC-MS: $m / z \mathrm{C}_{18} \mathrm{H}_{12} \mathrm{~N}_{2} \mathrm{~S}$ for $(\mathrm{M}+\mathrm{H})$ : Calculated: 288.07; Found, 289.0 (93.94\%). $\mathrm{IR} / \mathrm{cm}^{-1}: 1618.12,1579.39,1549.90,1460.04,1372.06$, $1275.19,1260.71,1162.74,1098.89,1010.64,868.12$, 750.70, 598.45 and 486.79. ${ }^{1} \mathrm{H} \mathrm{NMR}\left(\mathrm{CDCl}_{3}, 300 \mathrm{MHz}\right)$ : $9.17(1 \mathrm{H}, \mathrm{s}), 7.84-7.82(3 \mathrm{H}, \mathrm{m}), 7.77(1 \mathrm{H}, \mathrm{s}), 7.58(1 \mathrm{H}$, $\mathrm{d}, J=1.2 \mathrm{~Hz}), 7.55-7.52(2 \mathrm{H}, \mathrm{m}), 7.33-7.26(2 \mathrm{H}, \mathrm{m})$, $6.67(1 \mathrm{H}, \mathrm{s}) .{ }^{13} \mathrm{C} \mathrm{NMR}\left(\mathrm{CDCl}_{3}, 300 \mathrm{MHz}\right): 160.63,151.0$, $146.13,143.65,134.6,128.4,127.6,127.3,127.2,126.6$, $122.6,115.10,111.4,110.0 \mathrm{ppm}$.

2.2.17 2-(biphenyl-2-ylthio) quinazoline (Table 4, Entry q): Yellow thick mass [163 mg, Y: 85\%] $\mathrm{R}_{f}: 0.7,12 \%$ EtOAc/Pet ether; Analysis: LC-MS: $m / z \quad \mathrm{C}_{20} \mathrm{H}_{14} \mathrm{~N}_{2} \mathrm{~S}$ for $(\mathrm{M}+\mathrm{H})$ : Calculated: 314.09; Found, 315.0 (85.68\%). $\mathrm{IR} / \mathrm{cm}^{-1}: 1734.34,1616.75,1457.04,1435.81,1385.01$, $1370.63,1160.21,1095.67,755.44,697.46$ and $481.81 .{ }^{1} \mathrm{H}$ NMR $\left(\mathrm{CDCl}_{3}, 400 \mathrm{MHz}\right): 9.17$ (1H, s), 7.95 (1H, s), 7.847.78 (2H, m), 7.70-7.63 (3H, m), 7.57-7.51 (2H, m), 7.46$7.41(2 \mathrm{H}, \mathrm{m}), 7.38-7.29(1 \mathrm{H}, \mathrm{m}), 7.22-7.11(1 \mathrm{H}, \mathrm{m}), 6.77$ (1H, s). ${ }^{13} \mathrm{C} \mathrm{NMR}\left(\mathrm{CDCl}_{3}, 400 \mathrm{MHz}\right): 166.84,161.26$, $157.44,151.27,144.58,134.9,128.6,127.65,127.5,127.3$, $125.7,123.2,123.0,121.6,117,111.83 \mathrm{ppm}$.

2.2.18 Morpholine(4-(quinazolin-2-ylthio)phenyl)methanone (Table 4, Entry r): Pale yellow oil [Yield: $192 \mathrm{mg}$, 90\%] $\mathrm{R}_{f}$ : 0.6, 12\% EtOAc/Pet ether; Analysis: LC-MS: $m / z \mathrm{C}_{19} \mathrm{H}_{17} \mathrm{~N}_{3} \mathrm{O}_{2} \mathrm{~S}$ for $(\mathrm{M}+\mathrm{H})$ : Calculated: 351.10; Found, 
351.77 (92.77\%). IR $/ \mathrm{cm}^{-1}: 1765.18,1633.82,1557.88$, $1549.48,1434.67,1371.09,1278.81,1257.72,1162.29$, 1113.73, 1024.09, 761.32, 740.61, 692.14 and 492.15. ${ }^{1} \mathrm{H}$ NMR $\left(\mathrm{CDCl}_{3}, 300 \mathrm{MHz}\right): 9.15(1 \mathrm{H}, \mathrm{s}), 7.85-7.80(2 \mathrm{H}, \mathrm{m})$, 7.77-7.67 (3H, m), 7.56-7.52 (3H, m), 3.50-3.90 (8H, m). ${ }^{13} \mathrm{C} \mathrm{NMR}\left(\mathrm{CDCl}_{3}, 300 \mathrm{MHz}\right): 160.62,150.97,148.81$, $148.08,134.49,129.42,127.29,126.53,122.57,121.83$, $115.60,108.87,101.53,66.68,59.67 \mathrm{ppm}$.

\section{Results and Discussion}

The replacement of a multistep chemical synthesis with a transition metal catalyzed redox reaction is a powerful strategy to improve the efficiency and sustainability of synthesis. ${ }^{6,7}$ The potential of a transition metal catalyzed transformation is maximized when combined with straight-forward reaction conditions and the formation of widely utilized synthetic building blocks.

Recent work in the Chan, ${ }^{8}$ Cundy, ${ }^{9}$ and Evans ${ }^{10}$ laboratories have revealed the efficiency of copper(II) acetate in mediation of the cross-coupling of aryl boronic acids and phenols or amines to give biaryl ethers and aryl alkyl amines. We sought to develop a similar procedure for the formation of aryl alkyl sulfides and report here in the success of this approach. In general, this reaction (Scheme 1) proceeds as follows: Aryl boronic acids (2-2.2 equiv.) are allowed to react with a limiting quantity of quinazoline thiol (1.0 equiv.) with the mediation of copper(II) acetate and triethylamine in $\mathrm{CH}_{2} \mathrm{Cl}_{2}$ to give alkyl sulfides in $75-90 \%$ yield. The reaction proceeds facilely with a variety of aryl boronic acids. We set out to explore the scope of the method with respect to the substitution of the aryl ring (Table 4). In general, the reaction is unaffected by electronic factors but moderately affected by steric hindrance of the reaction center. We also examined the scope of the method with respect to the nature of the thiol nucleophile. These studies revealed that most thiols would enter into cross-coupling with phenyl boronic acid under the standard conditions (Table 3 ). Thus, this method can afford the diphenyl sulfide, the product of coupling of a primary thiol.

The functional group tolerance is good. The reaction proceeds with both electron-rich and electrondeficient aryl boronic acids, and works in the presence of additional functional groups including halides, esters, ethers, nitriles and aldehydes (Table 4, entries a-r).

Table 1. Optimization of the amine (base) for the cross coupling.

\begin{tabular}{|c|c|c|c|}
\hline \multirow[t]{2}{*}{ Entry } & \multirow[t]{2}{*}{ Base (Equiv) } & \multicolumn{2}{|c|}{${ }^{\mathrm{a}}$ Yield [\%] } \\
\hline & & $\overline{\mathrm{Cu}(\mathrm{OAc})_{2}}$ & $\mathrm{CuSO}_{4}$ \\
\hline 1 & Pyridine (1) & 40 & 25 \\
\hline 2 & Pyridine (5) & 65 & 45 \\
\hline 3 & $\mathrm{Et}_{3} \mathrm{~N}(2)$ & 75 & 50 \\
\hline b4 & $\mathrm{Et}_{3} \mathbf{N}$ & 88 & 60 \\
\hline 5 & DMAP (1) & 30 & 22 \\
\hline 6 & DMAP (5) & 45 & 30 \\
\hline 7 & $\mathrm{Cs}_{2} \mathrm{CO}_{3}(5)$ & 35 & 15 \\
\hline 8 & $\mathrm{Na}_{2} \mathrm{CO}_{3}(5)$ & 10 & 0 \\
\hline 9 & $\mathrm{~K}_{2} \mathrm{CO}_{3}(5)$ & 20 & 5 \\
\hline 10 & $\mathrm{CsF}(5)$ & 45 & 0 \\
\hline 11 & TBAF (5) & 52 & 22 \\
\hline 12 & KF (5) & 30 & 10 \\
\hline
\end{tabular}

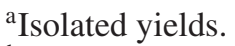

${ }^{\mathrm{b}}$ Reaction condition: triethyl amine (5 eq.), $\mathrm{Cu}(\mathrm{OAc})_{2}$ (Y: $88 \%$ ).

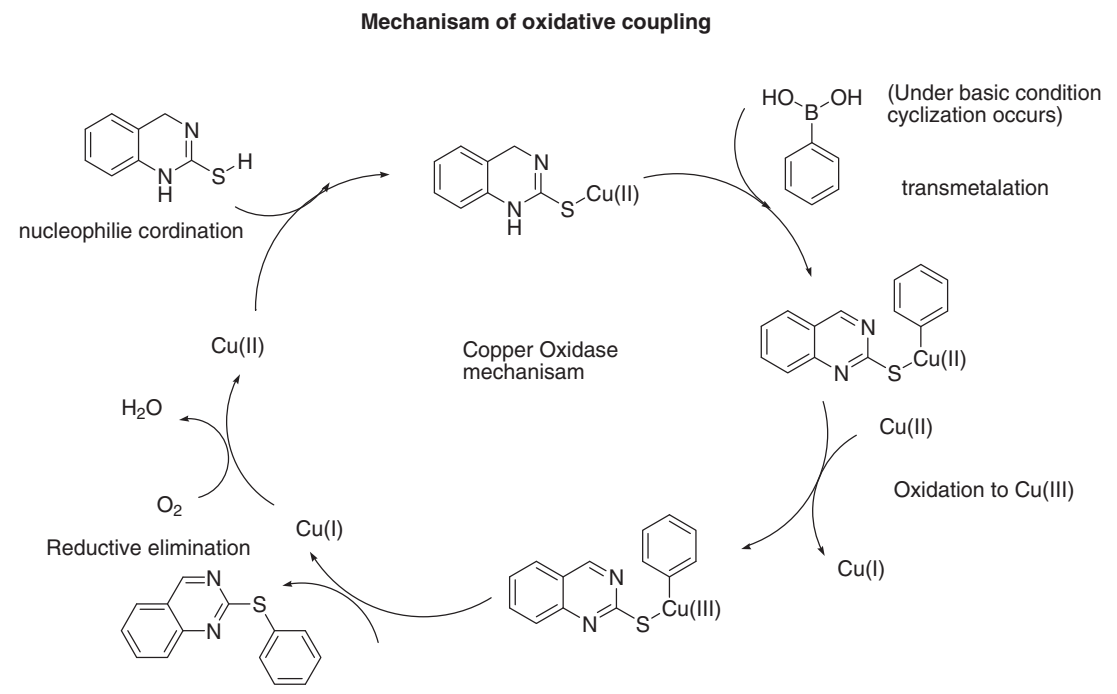

Scheme 1. Synthesis of $S$-aryl/heteroaryl-quinazoline derivatives through the cross-coupling of 1,4-dihydroquinazoline with various boronic acids. 
The most straightforward method for the synthesis of aryl/heteroaryl-thioquinazoline involves either cross-coupling of dihydro quinazoline with substituted boronic acid (Scheme 1) or a nucleophilic attack of aryl thiols by preformed thio-quinazoline.

To study the scope of the reaction in a further set of experiments, we examined the scope and generality of the approach for the cyclization followed by aromatization of 1,4-dihydro quinazoline with a series of alkyl and aryl boronic acids under optimum reaction conditions. Our results showed that both alkyl and aryl thiols can be efficiently converted to the corresponding cross-coupled products (Table 4).

Recently, we reported ${ }^{11}$ that copper catalyzed the formation of $S$-aryl quinazoline in a tandem onepot, three component reaction of 2-amino benzylamine, carbon disulfide and aryl halide. These results prompted us to investigate further C-S bond formation reactions of 2-thio-1,4-dihydroquinazoline with various aryl/heteroaryl boronic acids. The desired

Table 2. Evaluation of copper salts.

\begin{tabular}{lccc}
\hline Entry & Copper salt (Equiv) & Base (Equiv) & Yield [\%] \\
\hline 1 & $\mathrm{Cu}(\mathrm{OAc})_{2}(1.5)$ & Pyridine $(5.0)$ & 50 \\
2 & ${\mathrm{Cu}(\mathrm{OAc})_{2}(1.5)} \mathrm{Et}_{3} \mathrm{~N}(5.0)$ & 90 \\
3 & $\mathrm{CuCl}_{2}(1.5)$ & $\mathrm{Et}_{3} \mathrm{~N}(5.0)$ & 45 \\
4 & $\mathrm{CuCl}_{2}(1.5)$ & $\mathrm{Pyridine}(5.0)$ & 30 \\
5 & $\mathrm{CuSO}_{4}(1.5)$ & Pyridine $(5.0)$ & 50 \\
6 & $\mathrm{CuSO}_{4}(1.5)$ & $\mathrm{Et}_{3} \mathrm{~N}(5.0)$ & 70 \\
7 & $\mathrm{CuI}(1.5)$ & $\mathrm{Pyridine}^{(5.0)}$ & 45 \\
8 & $\mathrm{CuI}(1.5)$ & $\mathrm{Et}_{3} \mathrm{~N}(5.0)$ & 60 \\
9 & $\mathrm{Cu}(\mathrm{II})(1.5)$ & $\mathrm{Et}_{3} \mathrm{~N}(5.0)$ & 45 \\
10 & $\mathrm{Cu}(\mathrm{OAc})_{2}(1.5)$ & Pyridine/Et $_{3} \mathrm{~N}(5.0)$ & 65 \\
\hline
\end{tabular}

$S$-substituted quinazolines are obtained in excellent yields with short reaction times and a low catalytic amount of copper.

2-Aminobenzylamine can be transformed into dithiocarbamate by the reaction with carbon disulfide, then followed by cyclization to yield 1,4-dihydro quinazoline as a white solid. ${ }^{10}$ Dithiocarbamate is an important synthetic reagent to synthesize various biologically active heterocyclic compounds (Table 1). ${ }^{12-14}$

Optimization of reaction conditions for $\mathrm{Cu}$-catalyzed coupling of 2-thio-1,4-dihydroquinazolines and $p$-tolyl boronic acid is done. Our initial attempt to explore an effective catalytic system for the reaction in the presence of $\mathrm{CuSO}_{4}$ and pyridine under $\mathrm{N}_{2}$ atmosphere at room temperature gave yield of $26 \%$. We found that the removal of base, i.e., pyridine from the reaction mixture is difficult and tedious, which resulted in a lower yield of the product. Upon further screening for the conditions, fortunately, when we switched to copper acetate as catalyst, triethylamine as base and $\mathrm{CHCl}_{3}$ as solvent, the yield increased to $60 \%$. To our surprise, the yield could be significantly increased when changing the solvent to dichloromethane as the solvent. No significant improvement of the yield of the desired product 1a was observed when other copper salts, such as $\mathrm{CuCl}_{2}$ and $\mathrm{CuI}$ were used as the catalysts (Table 2, entries 3, 4 and 7,8 ). To improve the yield, different solvents were examined and $\mathrm{CH}_{2} \mathrm{Cl}_{2}$ gave the best yield compared with other solvents (Table 3, entries 1-14). Interestingly, when the reaction time was shortened to $8 \mathrm{~h}$, the yield increased to $88 \%$ (Table 4 ).

The experiment was performed with commercially available substituted boronic acids and $S$-1,4-dihydro

Table 3. Optimization reaction conditions in the copper-catalyzed coupling of thiol with substituted boronic acid.

\begin{tabular}{|c|c|c|c|c|}
\hline Entry & Base & Solvent & Catalyst & a'Yield [\%] \\
\hline 1 & Pyridine & $\mathrm{CCl}_{4}$ & $\mathrm{CuSO}_{4} .5 \mathrm{H}_{2} \mathrm{O}$ & No reaction \\
\hline 2 & $\mathrm{Et}_{3} \mathrm{~N}$ & $\mathrm{CCl}_{4}$ & $\mathrm{CuSO}_{4} .5 \mathrm{H}_{2} \mathrm{O}$ & No reaction \\
\hline 3 & Pyridine & $\mathrm{CCl}_{4}$ & $\mathrm{Cu}(\mathrm{OAc})_{2}$ & No reaction \\
\hline 4 & $\mathrm{Et}_{3} \mathrm{~N}$ & $\mathrm{CCl}_{4}$ & $\mathrm{Cu}(\mathrm{OAc})_{2}$ & No reaction \\
\hline 5 & Pyridine & $\mathrm{CHCl}_{3}$ & $\mathrm{CuSO}_{4} .5 \mathrm{H}_{2} \mathrm{O}$ & 42 \\
\hline 6 & $\mathrm{Et}_{3} \mathrm{~N}$ & $\mathrm{CHCl}_{3}$ & $\mathrm{CuSO}_{4} .5 \mathrm{H}_{2} \mathrm{O}$ & 55 \\
\hline 7 & Pyridine & $\mathrm{CHCl}_{3}$ & $\mathrm{Cu}(\mathrm{OAc})_{2}$ & 50 \\
\hline 8 & $\mathrm{Et}_{3} \mathrm{~N}$ & $\mathrm{CHCl}_{3}$ & $\mathrm{Cu}(\mathrm{OAc})_{2}$ & 60 \\
\hline 9 & Pyridine & $\mathrm{CH}_{2} \mathrm{Cl}_{2}$ & $\mathrm{CuSO}_{4} .5 \mathrm{H}_{2} \mathrm{O}$ & 55 \\
\hline 10 & $\mathrm{Et}_{3} \mathrm{~N}$ & $\mathrm{CH}_{2} \mathrm{Cl}_{2}$ & $\mathrm{CuSO}_{4} .5 \mathrm{H}_{2} \mathrm{O}$ & 40 \\
\hline 11 & Pyridine & $\mathrm{CH}_{2} \mathrm{Cl}_{2}$ & $\mathrm{Cu}(\mathrm{OAc})_{2}$ & 62 \\
\hline b 12 & $\mathbf{E t}_{\mathbf{3}} \mathbf{N}$ & $\mathrm{CH}_{2} \mathrm{Cl}_{2}$ & $\mathrm{Cu}(\mathrm{OAc})_{2}$ & 90 \\
\hline 13 & $\mathrm{Et}_{3} \mathrm{~N}$ & THF & $\mathrm{Cu}(\mathrm{OAc})_{2}$ & 55 \\
\hline 14 & $\mathrm{Et}_{3} \mathrm{~N}$ & DMF & $\mathrm{Cu}(\mathrm{OAc})_{2}$ & 65 \\
\hline
\end{tabular}

asolated yields.

${ }^{\mathrm{b}}$ Reaction condition: triethyl amine (5 eq.), $\mathrm{Cu}(\mathrm{OAc})_{2}$ (1.5 eq.) DCM. (Y: 90\%). 
Table 4. Substituent effects in the cross coupling of thiol with substituted boronic acid.

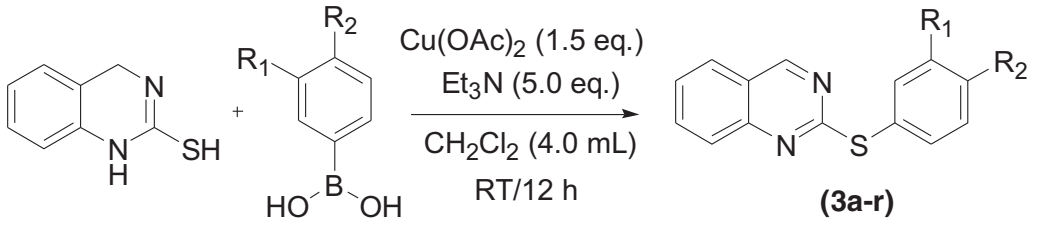

(2) (2.0 eq.)

18 examples

$75-90 \%$ yield

$\mathrm{R}_{1}$ and $\mathrm{R}_{2}=\mathrm{H}, \mathrm{Cl}, \mathrm{Br}, \mathrm{CH}_{3}, \mathrm{CF}_{3}, \mathrm{OCF}_{3}$,

heterocyclic, biphenyl and morpholine

Entry 1,4-dihydroquinazoline-2-thiol (1) Boronic acid (2)

Product $^{\mathrm{b}}(3)$

Yield $^{\mathrm{a}}(\%)$

a<smiles>SC1=NCc2ccccc2N1</smiles>

$\mathrm{HO}_{\mathrm{B}^{-}} \mathrm{OH}$

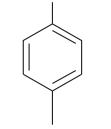<smiles>Ic1ccc(Sc2ncc3ccccc3n2)cc1</smiles>

b<smiles>SC1=NCc2ccccc2N1</smiles>

$\mathrm{HO}_{\substack{\mathrm{B} \\ \mathrm{O}}}^{\mathrm{OH}^{2}}$<smiles>C=CCSc1ncc2ccccc2n1</smiles>

$\mathrm{HO}^{-}{ }^{-} \mathrm{OH}$<smiles>SC1=NCc2ccccc2N1</smiles><smiles>Cc1ccc(C(C)C)cc1</smiles>

$\mathrm{HO}_{\mathrm{B}^{-}}-\mathrm{OH}$

d<smiles>SC1=NCc2ccccc2N1</smiles><smiles>c1ccncc1</smiles><smiles>c1ccc2nc(Sc3ccncc3)ncc2c1</smiles>

$\mathrm{HO}_{{ }_{\mathrm{B}}}-\mathrm{OH}$<smiles>SC1=NCc2ccccc2N1</smiles><smiles>COc1cccc(O)c1</smiles><smiles>COc1cccc(Sc2ncc3ccccc3n2)c1</smiles>

86

e<smiles>SC1=NCc2ccccc2N1</smiles><smiles>O=[N+]([O-])c1cccc(B(O)O)c1</smiles><smiles>CC(C)c1ccc(Sc2ncc3ccccc3n2)cc1</smiles>

f<smiles>SC1=NCc2ccccc2N1</smiles><smiles>O=Cc1ccc(B(O)O)s1</smiles><smiles>O=[N+]([O-])c1cccc(Sc2ncc3ccccc3n2)c1</smiles>

g<smiles>SC1=NCc2ccccc2N1</smiles><smiles>OB(O)c1cc2ccccc2s1</smiles><smiles>O=Cc1ccc(Sc2ncc3ccccc3n2)s1</smiles>

h<smiles>SC1=NCc2ccccc2N1</smiles><smiles>OB(O)c1cccc(Cl)c1Cl</smiles><smiles></smiles>

i<smiles>SC1=NCc2ccccc2N1</smiles><smiles>OB(O)c1cnc2ccccc2c1</smiles><smiles></smiles> 
Table 4. (continued)

\begin{tabular}{|c|c|c|c|c|}
\hline Entry & 1,4-dihydroquinazoline-2-thiol (1) & Boronic acid (2) & Product $^{\mathrm{b}}(3)$ & Yield $^{\mathrm{a}}(\%)$ \\
\hline k & $\mathrm{H}$ & & & 90 \\
\hline 1 & $\mathrm{H}$ & 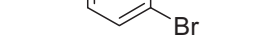 & & 82 \\
\hline m & & $\mathrm{HO}^{-5}$ & & 76 \\
\hline n & 511 & & & 72 \\
\hline о & & $\mathrm{CF}_{3}$ & & 80 \\
\hline p & SH & & & 90 \\
\hline $\mathbf{q}$ & UTI & & & 85 \\
\hline $\mathbf{r}$ & SH & $\mathrm{OH}$ & & 90 \\
\hline
\end{tabular}

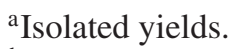

${ }^{\mathrm{b}}$ All the compounds were characterized by IR, ${ }^{1} \mathrm{H}$ NMR, ${ }^{13} \mathrm{C}$ NMR spectroscopy and mass spectrometry.

quinazolines by using $\mathrm{Cu}(\mathrm{OAc})_{2}, \mathrm{Et}_{3} \mathrm{~N}$ as a base in dichloromethane at r.t. for $12 \mathrm{~h}$. In this reaction, aromatization on 1,4-dihydroquinazolines is followed by $S$-alkylation. This is a novel route for this synthesis. The desired $S$-alkyl quinazolines was isolated in good yields. In this, several boronic acids such as aromatic, substituted aromatic, heterocyclic compounds were screened to optimize the method. It was observed that using $\mathrm{Cu}(\mathrm{OAc})_{2}, \mathrm{Et}_{3} \mathrm{~N}$ in dichloromethane gave the best results under normal conditions. Reactions occurred with good yields, broad scope, and high tolerance of functional groups.

We have reported an efficient synthesis of a C-S bond formation via aromatization in normal argon atmospheric conditions. We have reported a general synthetic protocol for the formation of aryl-sulfur bonds, using copper(II) catalysts. The optimized protocol tolerates a variety of electron-donating and electronwithdrawing groups on the aryl boronic acids. The scope and generality of this method were later harnessed to synthesize a range of other analogues, listed in Table 4 .

\section{Conclusions}

In conclusion, a new method for the cross-coupling of heteroaromatic thioethers with boronic acids has been described. The readily available starting materials and mild and neutral reaction conditions suggest that this procedure could find wide applicability in synthesis and drug design. 


\section{Supplementary Information (SI)}

NMR, IR and mass spectra of the synthesized compounds are reported in Supplementary Information, available at www.ias.ac.in/chemsci.

\section{Acknowledgements}

Pulakhandam S K is thankful to the authorities of Jawaharlal Nehru Technological University Kakinada for registration and GITAM University Hyderabad for providing laboratory facilities.

\section{References}

1. (a) Herz W, Grisebach H, Kirby G W and Tamm C 1984 In Progress in the Chemistry of Organic Natural Products S Johne (Ed.) (Wien: Spinger-Verlag) 46 p.159; (b) Brossi A 1986 The Alkaloids, Chemistry and Pharmacology S Johne (Ed.) (New York: Academic Press) 29 p. 99

2. (a) Yudin A K and Hartwig J F 2010 Catalyzed Carbon-Heteroatom Bond Formation (Weinheim: Wiley VCH); (b) Hartwig J F 1998 Carbon-Heteroatom BondForming Reductive Eliminations of Amines, Ethers, and Sulfides Acc. Chem. Res. 31 852; (c) Hartwig J F 1998 Transition metal catalyzed synthesis of arylamines and aryl ethers from aryl halides and triflates: Scope and Mechanism Angew. Chem. Int. Ed. 37 2046; (d) Ley S V and Thomas A W 2003 Modern synthetic methods for copper mediated C(aryl)[bond]O, C(aryl)[bond]N, and C(aryl)[bond]S bond formation Angew. Chem. Int. Ed. 42 5400; (e) Beccalli E M, Broggini G, Martinelli M and Sottocornola S 2007 C-C, C-O, C-N bond formation on SP2 Carbon by $\mathrm{Pd}$ (II)-catalyzed reactions involving oxidant agents Chem. Rev. 107 5318; (f) Monnier F and Taillefer M M 2009 Catalytic C-C, C-N, and C-O Ullmann-type coupling reactions Angew. Chem. Int. Ed. 486954

3. (a) Bernardi F, Csizmadia I G and Mangini A 1985 In Organic Sulfur Chemistry: Theoretical and Experimental Advances (Amsterdam: Elsevier) 19; (b) De Martino G, Edler M C, La Regina G, Coluccia A, Barbera M C, Barrow D, Nicholson R I, Chiosis G, Brancale A, Hamel E, Artico M and Silvestri R 2006 New arylthioindoles: Potent inhibitors of tubulin polymerization. 2. Structure-activity relationships and molecular modeling studies J. Med. Chem. 49 947; (c) Gangjee A, Zeng Y, Talreja T, McGuire J J, Kisliuk R L and Queener S F 2007 Design and synthesis of classical and non-classical 6-arylthio-2,4-diamino5-ethylpyrrolo[2,3-d]pyrimidines as antifolates $\mathrm{J}$. Med. Chem. 503046

4. (a) Tiecco M 1988 Selective Dealkylations of Aryl Alkyl Ethers, Thioethers and Selenoethers Synthesis 10 749; (b) Thuillier A and Metzner P 1994 Sulfur Reagents in Organic Synthesis: Tellurium-containing Heterocycles (New York: Academic Press)

5. (a) Kondo $\mathrm{T}$ and Mitsudo $\mathrm{T} 2000$ Metal catalyzed carbon-sulfur bond formation Chem. Rev. 100 3205; (b) Correa A, Carril M and Bolm C 2008 Iron catalyzed S-arylation of thiols with aryl iodides Angew. Chem. Int. Ed. 47 2880; (c) Gangjee A, Zeng Y, Talreja T, McGuire J J, Kisliuk R L and Queener S F 2007 Design and synthesis of classical and non classical 6-arylthio-2,4-diamino-5-ethyl pyrrolo[2,3-d]pyrimidines as antifolates J. Med. Chem. 503046

6. (a) Tietze L F 1996 Domino Reactions in organic synthesis Chem. Rev. 96 115; (b) Tietze L F and Rackelmann N 2004 Domino reactions in the synthesis of heterocyclic natural products and analogs Pure Appl. Chem. 76 1967; (c) Wasilke J C, Obrey S J, Baker R T and Bazan G C 2005 Concurrent tandem catalysis Chem. Rev. 105 1001; (d) Sunderhaus J D and Martin S F 2009 Applications of Multicomponent Reactions to the Synthesis of Diverse Heterocyclic Scaffolds Chem. Eur. J. 151300

7. Hua-Jian X, Yong-Qiang Z, Teng F and Yi-Si F 2012 Chan-Lam-Type S-Arylation of Thiols with Boronic Acids at Room Temperature J. Org. Chem. 772878

8. (a) Chan D M T, Monaco K L, Wang R P and Winters M P 1998 New N- and O-arylations with phenyl boronic acids and cupric acetate Tetrahedron Lett. 39 2933; (b) Lam P Y S, Clark C G, Saubern S, Adams J, Winters M P, Chan D M T and Combs A 1998 New aryl/heteroaryl $\mathrm{C}-\mathrm{N}$ bond cross coupling reactions via arylboronicacid/cupric acetae arylation Tetrahedron Lett. 392941

9. Cundy D J and Forsyth S A 1998 Cupric Acetate Mediated N-Arylation by Arylboronic Acids: A Preliminary Investigation into the Scope of Application Tetrahedron Lett. 397979

10. Evans D A, Katz J L and West T R 1998 Synthesis of Diaryl ethers through the copper promoted Arylation of phenols with Aryl boronicacids: An Expedient synthesis of Thyroxine Tetrahedron Lett. 392937

11. Satya K P, Ramana M R V V, Reddy M R P, Naresh K K and Srinivas K 2016 Tandem one pot (Top) and efficient method for the palladium reagent-catalyzed cross coupling of quinazoline thiols J. Het. Chem. 53784

12. Parthasarathi D, Kiran Kumar C, Naresh K K, Md Innus, Javed I and Srinivas N 2008 Dithiocarbamate and CUO promoted one-pot synthesis of 2-(N-substituted)aminobenzimidazoles and related heterocycles Tetrahedron Lett. 49992

13. Naresh K K, Sreeramamurthy K, Sadananda P, Mukkanti K and Parthasarathi D 2010 Dithiocarbamate and DBU promoted amide bond formation under microwave condition Tetrahedron Lett. 51899

14. Naresh K K, Venkatanarayana M and Srinivas K 2015 Dithiocarbamate promoted practical Synthesis of N-Aryl-2-aminobenzazoles: Synthesis of novel AuroraA kinase inhibitor J. Chem. Sci. 127447 